'Departamento de Cirugía y Traumatología, Universidad de La Frontera.

${ }^{2}$ Centro de Investigación en Ciencias Biomédicas, Universidad Autónoma de Chile.

${ }^{3}$ Alumno del Programa de Doctorado en Ciencias Médicas, Universidad de La Frontera.

${ }^{4}$ Departamento de

Psicología, Universidad

Autónoma de Chile.

${ }^{a}$ Alumno del Programa de Doctorado en Ciencias Médicas, Universidad de La Frontera.

Parcialmente financiado por proyecto DI120037 de la Dirección de Investigación Universidad de La Frontera.

Recibido el 8 de octubre de 2012, aceptado el 1 de mayo de 2013.

Correspondencia a:

Dr. Carlos Manterola Departamento de Cirugía y Traumatología, Universidad de La Frontera.

Casilla 54-D, Temuco, Chile.

Teléfonos: 56-45-32576

Fax: 56-45-325761

E-mail: carlos.manterola@ ufrontera.cl

\title{
Uso versus no uso de malla en hernioplastía inguinal: ¿existe un rol para la reparación sin malla? Revisión global de la evidencia
}

\author{
CARLOS MANTEROLA ${ }^{1,2}$, SEBASTIÁN URRUTIA ${ }^{3}$, TAMARA OTZEN ${ }^{4, a}$
}

\section{Effectiveness of mesh inguinal hernia repair. A systematic review}

There is controversy about the effectiveness of mesh techniques for inguinal hernia repair (IHR) when compared with non-mesh procedures. The aim of this study was to systematically review the available evidence on the effectiveness of mesh compared to non-mesh IHR techniques. Cochrane Central and MEDLINE databases and the TRIP Database engine were reviewed looking for systematic reviews (SR) and clinical trials (CT) published from 1990 to 2012, comparing the effectiveness, in terms of operative time, postoperative pain, morbidity, hospital stay, return to work, costs and recurrence, of mesh and non-mesh IHR techniques. We retrieved 3,069 articles, 17 of which met the selection criteria ( 3 SR and $14 R C T$ ), representing a population of 15,124 subjects (7361 mesh and 7763 non-mesh IHR). According to these studies, mesh IHR techniques are associated with less surgical time, pain, hospital stay, recurrence and sooner return to work than non-mesh techniques. It is concluded that mesh techniques are more effective than those not using a mesh.

(Rev Med Chile 2013; 141: 932-939). Mesh.

Key words: Evidence-Based Medicine; Hernia Inguinal; Herniorrhaphy; Surgical

E xiste evidencia que sugiere que el riesgo estimado de tener una hernia inguinal es $27 \%$ para hombres y $3 \%$ para mujeres ${ }^{1}$; y que las tasas anuales de morbilidad asociadas al diagnóstico varían de 100 a 300 por 100.000 habitantes ${ }^{2}$.

No obstante ello, existe controversia respecto de las opciones de hernioplastía inguinal (HI), y recién en 2009 se publicó por parte de la European Hernia Society, una guía para el tratamiento de la hernia inguinal, en la que se recomiendan preferentemente las técnicas de Lichtenstein y Shouldice ${ }^{3}$.

A pesar de ser una cirugía frecuente, problemas postoperatorios menores como desarrollo de seroma, hematoma, etc., no son infrecuentes; y complicaciones mayores como lesión del cordón, vena o arteria femoral, o nervios ilioinguinal o genitofemoral son ocasionales ${ }^{4}$. Sin embargo, el principal inconveniente es la recurrencia ${ }^{4}$; con cifras de $5 \%$ a $15 \%{ }^{5}$.

Por lo anterior, es que el concepto de reparación abierta "sin tensión" con malla (Lichtenstein 1989) se ha hecho popular ${ }^{6}$. Supuestamente es técnicamente sencillo, se puede realizar de forma ambulatoria, con anestesia local, se asocia a menor dolor y permite rápido retorno laboral ${ }^{6,7}$. Sin embargo, persiste la controversia acerca de la efectividad de las técnicas de HI con malla vs sin malla.

El objetivo de este estudio es evaluar la efectividad de las técnicas de HI con malla en comparación con las técnicas de HI sin utilización de malla. 


\section{Material y Método}

Diseño: Revisión global de la evidencia.

Población: Revisiones sistemáticas de la literatura [RS] y ensayos clínicos con asignación aleatoria [EC]) publicados desde enero de 1990 a agosto de 2012, sin restricción idiomática; que incluyeran población mayor de 18 años; y evaluaran y compararan la efectividad de la reparación quirúrgica de la hernia inguinal con malla versus sin malla. Se excluyeron artículos de casuística contaminados con pacientes intervenidos de urgencia o por vía laparoscópica.

Metodología de búsqueda: Se realizó según componentes PICoR: población de interés $(\mathrm{P})$, intervención que se desea valorar (I), el comparador para la intervención en estudio (Co), y variable resultado que se medirá a partir de la intervención en estudio (R). A partir de esta estrategia, se buscaron estudios respecto de sujetos con hernia inguinal primaria $(\mathrm{P})$, a los que se realizó una HI sin malla (I), que se compararon con sujetos similares a los que se les realizó una HI con malla (Co), y cuyas variables de respuesta fuesen: dolor postoperatorio, morbilidad, recurrencia, etc. (R). Para ello, se revisaron las siguientes bases de datos: Cochrane Central Register of Controlled Trials, MEDLINE (a través del buscador PubMed) y el motor de búsqueda TRIP Database. Se realizaron búsquedas sensibles utilizando términos $\mathrm{MeSH}$, palabras libres y los conectores booleanos AND y OR, utilizando estrategias que se adaptaron para cada base de datos. De este modo, se generaron los siguientes comandos: "hern* in Record Title not lumbar not disc*, para Cochrane Central Register of Controlled Trials; ((inguinal hernia [Title]) OR herniorrhaphy [Title]) OR hernioplasty [Title]) OR hernias [Title]) OR hernia repair [Title]) OR hernia [MeSH Terms]) NOT lumbar [Title]) NOT discal [Title]) NOT diaphragmatic NOT disc NOT perineal), con los límites: "Publication date from 1967 to 2012, Humans, Systematic Reviews, Review, Randomized Controlled Trial, Meta-Analysis, Clinical Trial, Adult: 19+ years", para MEDLINE; y finalmente, el comando "title:hernia or herniorrhaphy or hernia repair" and (not title: lumbar or discal or disc), para TRIP Database.

Sintesis y valoración de la evidencia: Se comenzó con una evaluación del riesgo de sesgo (validez interna) de los estudios; para lo cual, se generaron tablas de resumen ${ }^{8}$, se aplicó Escala de
Jadad $^{9,10}$, y construyeron tablas de validez (utilizando diseño propuesto por el SIGN ${ }^{11}$. Posteriormente, se realizó una clasificación de niveles de evidencia, utilizando el sistema propuesto por el Oxford Centre for Evidence Based Medicine ${ }^{12}$. Finalmente, se realizó una clasificación global de la evidencia para cada intervención, la que se agrupó en: Alta, moderada y baja; aplicando un enfoque modificado del sistema GRADE, que considera el diseño del estudio, el riesgo de sesgo, la coherencia, la precisión de los resultados, el sesgo de publicación y si la evaluación de la evidencia es directa o indirecta ${ }^{13}$.

Variables de medición: Las variables consideradas fueron: tiempo quirúrgico, dolor postoperatorio inmediato, morbilidad postoperatoria general, morbilidad postoperatoria específica (incidencia de seroma, hematoma e infección del sitio operatorio [ISO]), tiempo de hospitalización, retorno a la actividad laboral, costes totales involucrados, dolor postoperatorio persistente y recurrencia.

Plan de análisis: Los datos seleccionados se recolectaron en una planilla Windows Excel. Se eliminaron aquellos EC que estaban incluidos en alguna RS. Posteriormente, se utilizó el paquete estadístico STATA 10.0, aplicando estadística descriptiva, con cálculo de medidas de tendencia central y dispersión.

Aspectos éticos: Durante el análisis de los artículos seleccionados se realizó enmascaramiento de los autores y centros de estudio, maniobra que permitió además reducir sesgos de selección y análisis.

\section{Resultados}

Se recuperaron 3.069 artículos y 17 de ellos que cumplían con los criterios de selección fueron finalmente incluidos, 3 RS y 14 EC (Figura 1). Las 3 RS fueron obtenidas a partir de la base de datos Cochrane Library ${ }^{14-16}$. Estas incluyen 56 EC en los que se estudia un total de 11.806 pacientes (5.702 con HI con malla y 6.104 con HI sin malla) Respecto de los EC, uno se obtuvo de Cochrane Central Register of Controlled Trials ${ }^{17}, 12$ a partir de MEDLINE ${ }^{18-29}$, uno desde TRIP Database ${ }^{30}$; estos representan una población de 3.318 pacientes ( 1.659 con malla y 1.659 sin malla). Por lo anteriormente expuesto, los resultados que se exponen 


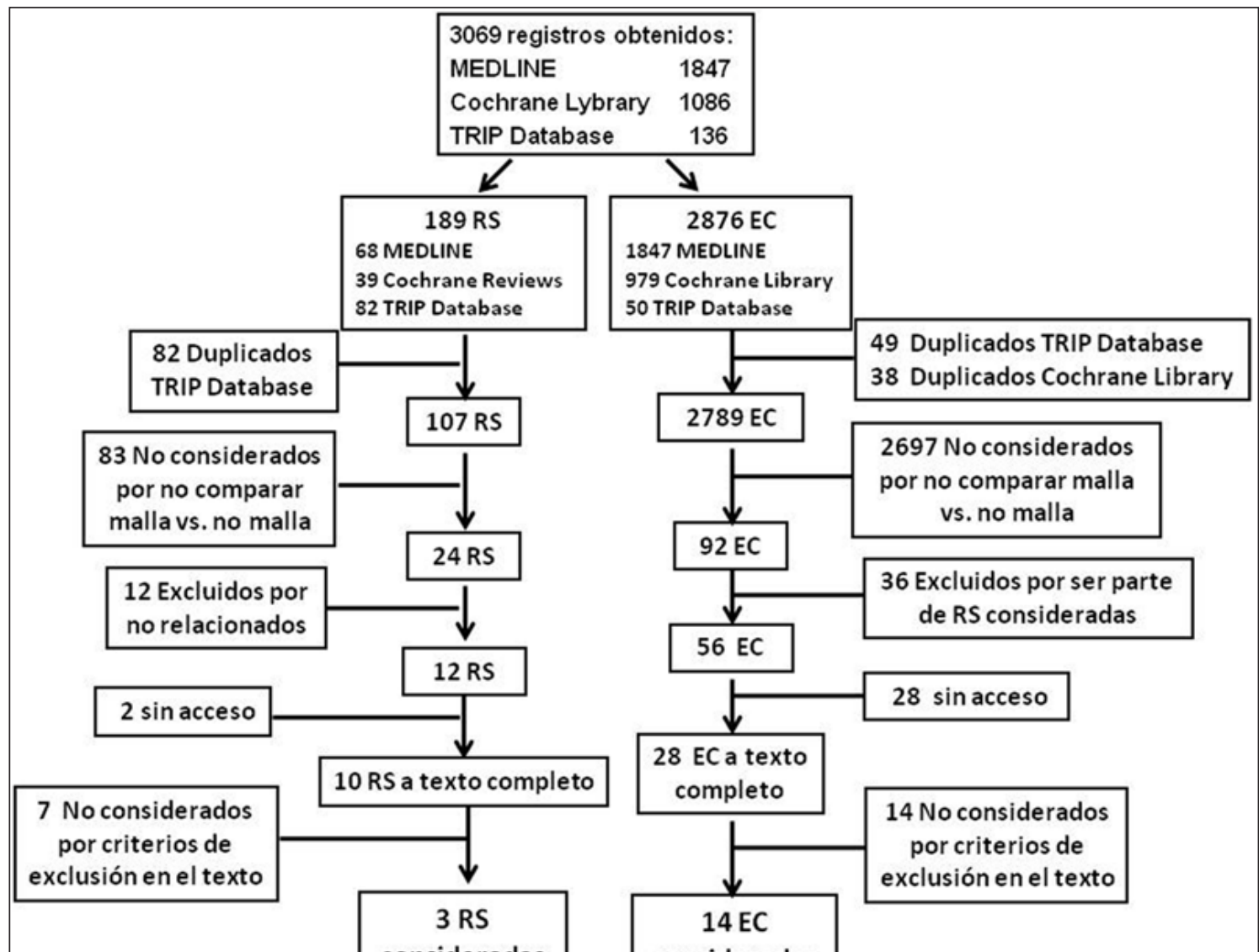

Figura 1. Diagrama de flujo de los resultados de la búsqueda realizada según la metodología descrita.

a continuación de basan en datos provenientes de una población total 15.124 sujetos intervenidos por hernia inguinal, $7.361 \mathrm{HI}$ con malla y 7.763 HI sin malla.

Las técnicas evaluadas para HI con malla fueron mayoritariamente Lichtenstein y dos EC en los que se utilizaron las técnicas de Stoppa y de Robins (uno de cada cual). Las técnicas evaluadas para $\mathrm{HI}$ sin malla fueron Shouldice (66\%), Bassini (20\%) y otras (14\%, entre las que se cuentan las técnicas de McVay, Desarda o Pérez-Fontana, Maloney darn y Rutkow). A continuación se detalla la síntesis de los hallazgos encontrados, cuyo resumen incluyendo la calidad de la evidencia se puede obvervar en la Tabla 1.

\section{Tiempo quirúrgico}

Se localizaron 8 artículos (2 RS, 6 EC) en los que se comparó la variable "duración de la cirugía"; la que fue definida como el tiempo en minutos dentro del quirófano. En una RS en la que se analizó esta variable con 5 EC y 1.251 pacientes, verificó que la duración de cirugía fue menor en las técnicas con malla respecto de las sin malla en 9,6 minutos ${ }^{16}$ (estudio de tratamiento 1a). En $3 \mathrm{EC}$ se verificó un menor tiempo quirúrgico para $\mathrm{HI}$ con malla ${ }^{22-24}$; cuyo impacto fue de $33,1 \pm 11,1 \mathrm{~min}$ vs $49,1 \pm 8,1$ min para HI con y sin malla respectivamente $(\mathrm{p}<0,05)^{23}$; $\mathrm{y}$ de $38,5 \pm 5,1$ min vs 40,4 $\pm 3,3$ min para HI con y sin malla respectivamente $(\mathrm{p}<0,01)^{22}$; (estudios de tratamiento $1 b$ y $2 b$ ). Por otra parte, se identificó una RS ${ }^{15}$ (compuesta por $20 \mathrm{EC}$ ) en la que se observó que las técnicas con malla tomaron en promedio 7 a 10 min menos que la técnica de Shouldice, pero 1 a 4 min más que otros procedimientos sin malla, lo que representa gran heterogeneidad (estudio de tratamiento 1a); por otra parte, en $3 \mathrm{EC}^{17,19,28}$, no se evidenciaron 
Uso vs no uso de malla en hernioplastía inguinal. Overview - C. Manterola et al

Tabla 1. Tabla de resumen de la evidencia

\begin{tabular}{|c|c|c|c|}
\hline $\begin{array}{l}\text { Variables } \\
\text { estudiadas }\end{array}$ & $\begin{array}{l}\text { n de } \\
\text { estudios }\end{array}$ & $\begin{array}{l}\text { Calidad de } \\
\text { la evidencia }\end{array}$ & Resumen de los hallazgos \\
\hline \multirow[t]{2}{*}{$\begin{array}{l}\text { Tiempo } \\
\text { quirúrgico }\end{array}$} & $\begin{array}{l}2 \mathrm{RS} \\
1 \mathrm{EC}\end{array}$ & Alta & Menor duración en técnicas de $\mathrm{HI}$ con malla respecto de las sin malla \\
\hline & $5 \mathrm{EC}$ & Moderada & $\begin{array}{l}\text { Menor duración en técnicas de } \mathrm{HI} \text { con malla o sin diferencias signifi- } \\
\text { cativas }\end{array}$ \\
\hline $\begin{array}{l}\text { Dolor postop. } \\
\text { inmediato }\end{array}$ & $\begin{array}{l}1 \mathrm{RS} \\
9 \mathrm{EC}\end{array}$ & Moderada & $\begin{array}{l}\text { Significativamente menor en las técnicas con malla; o no hubo diferen- } \\
\text { cias significativas }\end{array}$ \\
\hline $\begin{array}{l}\text { Morbilidad } \\
\text { postop. general }\end{array}$ & $6 \mathrm{EC}$ & Moderada & $\begin{array}{l}\text { Sin diferencias significativas en morbilidad general, incidencia de seroma, } \\
\text { hematoma ni de infección del sitio operatorio }\end{array}$ \\
\hline \multirow[t]{2}{*}{$\begin{array}{l}\text { Tiempo de } \\
\text { hospitalización }\end{array}$} & $\begin{array}{l}2 \text { RS } \\
3 \mathrm{EC}\end{array}$ & Alta & Menor estadía hospitalaria postoperatoria en $\mathrm{HI}$ con malla \\
\hline & $\begin{array}{l}1 \mathrm{RS} \\
3 \mathrm{EC}\end{array}$ & Moderada & Sin diferencias significativas \\
\hline \multirow[t]{2}{*}{$\begin{array}{l}\text { Retorno a la } \\
\text { actividad laboral }\end{array}$} & $\begin{array}{l}1 \mathrm{RS} \\
3 \mathrm{EC}\end{array}$ & Alta & $\begin{array}{l}\text { Pacientes sometidos a } \mathrm{HI} \text { con malla regresaron a sus actividades cotidia- } \\
\text { nas antes que aquellos sometidos a } \mathrm{HI} \text { sin malla }\end{array}$ \\
\hline & $\begin{array}{l}1 \mathrm{RS} \\
4 \mathrm{EC}\end{array}$ & Moderada & $\begin{array}{l}\text { Regresaron antes, pero con diferencias marginales o sin diferencias } \\
\text { significativas }\end{array}$ \\
\hline \multirow{2}{*}{$\begin{array}{l}\text { Costes } \\
\text { involucrados }\end{array}$} & $1 \mathrm{EC}$ & Alta & Costo total menor para el grupo de $\mathrm{HI}$ con malla respecto de Shouldice \\
\hline & $4 \mathrm{EC}$ & Moderada & $\begin{array}{l}\text { Costos asociados a la reparación quirúrgica fueron significativamente } \\
\text { menores en el grupo de pacientes con } \mathrm{HI} \text { sin malla }\end{array}$ \\
\hline \multirow[t]{2}{*}{$\begin{array}{l}\text { Dolor postop. } \\
\text { persistente }\end{array}$} & $\begin{array}{l}2 \mathrm{RS} \\
1 \mathrm{EC}\end{array}$ & Alta & Menor dolor crónico en pacientes con reparación con malla \\
\hline & $\begin{array}{l}1 \mathrm{RS} \\
8 \mathrm{EC}\end{array}$ & Moderada & No se objetivaron diferencias significativas \\
\hline \multirow[t]{2}{*}{ Recurrencia } & $\begin{array}{l}3 \mathrm{RS} \\
5 \mathrm{EC}\end{array}$ & Alta y moderada & Significativamente menor en las técnicas de HI con malla \\
\hline & $3 \mathrm{EC}$ & Moderada & Sin diferencias significativas \\
\hline
\end{tabular}

Alta calidad: es muy poco probable que futuros estudios cambien la confianza en la estimación del efecto y por lo tanto, la confianza es alta. Moderada calidad: es probable que futuros estudios cambien la confianza en la estimación del efecto.

diferencias estadísticamente significativas para algún tipo de técnica (estudios de tratamiento $2 b$ ).

\section{Dolor postoperatorio inmediato}

Se localizaron 10 artículos (1 RS y 9 EC) en los que se comparó la variable "dolor postoperatorio inmediato". En cuatro artículos, una $\mathrm{RS}^{14} \mathrm{y}$ $3 \mathrm{EC}^{23-25}$, el dolor postoperatorio fue significativamente menor en las técnicas con malla (estudios de tratamiento $2 a$ y $2 b$ ); de hecho el impacto de este resultado se reporta de forma clara en dos de ellos en los que se constató un EVA de 3,0 en HI con malla vs 5,0 en HI sin malla $(\mathrm{p}<0,01)^{23}$ y un EVA de 1,8 en HI con malla vs 2,8 en HI sin malla $(\mathrm{p}<0,05)^{24}$ (estudios de tratamiento $2 b$ ). Por otra parte, se identificaron $6 \mathrm{EC}^{17-19,21,22,28}$ en los que no se verificaron diferencias estadísticamente significativas al comparar ambas técnicas respecto de esta variable (estudios de tratamiento $2 b$ ).

\section{Morbilidad postoperatoria en general}

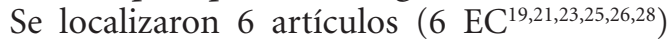
en los que se comparó la variable "morbilidad postoperatoria general", tanto las complicaciones postoperatorias tempranas como tardías sin encontrar diferencias significativas (estudios de tratamiento $2 b$ ). Al estudiar las principales causas de morbilidad (seroma, hematoma e ISO) por separado; se logró evidenciar lo siguiente:

Para el desarrollo de seroma se identificaron 7 
artículos. En 6 de ellos ( $3 \mathrm{RS}^{14-16}$ y $3 \mathrm{EC}^{19,21,28}$ ), no se encontraron diferencias significativas (estudios de tratamiento $2 a$ y $2 b$ ); y en el EC restante ${ }^{26}$, en el que se comparó las técnicas de Lichtenstein con la de Dasarda, no hubo diferencias a los siete días de la cirugía, sin embargo, el porcentaje de seroma fue mayor en el grupo inervenido con técnica de Lichtenstein a los 30 días de seguimiento, aunque la diferencia fue marginal (estudios de tratamiento $2 b)$.

Para el desarrollo de hematoma se identificaron 4 artículos. En $2 \mathrm{RS}^{14,15}$ y un $\mathrm{EC}^{21}, 2010$ ) no se verificaron diferencias estadísticamente significativas entre ambos grupos de tratamiento (estudios de tratamiento $1 a, 2 a$ y $2 b$ ); y en otra $\mathrm{RS}^{16}$, la frecuencia de hematoma fue mayor en el grupo de Shouldice, aunque la diferencia fue marginal, con un OR de 0,63 y un IC de $95 \%$ de 0,24 a 1,66 (estudios de tratamiento 1a).

Finalmente, para el desarrollo de seroma se identificaron 8 artículos. En 7 de ellos $\left(2 \mathrm{RS}^{14,15}\right.$ y $\left.5 \mathrm{EC}^{17,19,21,25,28}\right)$, no se observaron diferencias significativas (estudios de tratamiento $1 a, 2 a$ y $2 b$ ); sin embargo, una $\mathrm{RS}^{16}$, reportó una menor incidencia (aunque marginal) de ISO en los pacientes intervenidos con técnica de Shouldice, con un OR de 0,74 y un IC de $95 \%$ de 0,37 a 1,49 (estudios de tratamiento 1a).

\section{Tiempo de hospitalización}

Se localizaron 8 artículos en los que se comparó la variable "tiempo de hospitalización" (3 RS y 5 EC). En dos $\mathrm{RS}^{14,15}$ y un $\mathrm{EC}^{25}$, se evidenció menor estadía hospitalaria postoperatoria en sujetos a $\mathrm{HI}$ con malla (estudios de tratamiento $1 a, 2 a$ y $2 b$ ); de hecho, en este $\mathrm{EC}^{25}$ en que se compara Lichtenstein con Bassini, la estancia media fue de 4,5 días vs 7,6 días respectivamente, con un valor de $\mathrm{p}<0,01$ (estudios de tratamiento $2 b$ ). En otro $\mathrm{EC}^{17}$, también se objetivó menor estadía hospitalaria, aunque no significativa, en el grupo de pacientes de HI de Lichtenstein respecto de HI Bassini modificada (4,97 días vs 5,7 días respectivamente), (estudios de tratamiento $2 b$ ). Por otra parte, en una $\mathrm{RS}^{16}$ $\mathrm{y}$ tres $\mathrm{EC}^{18,19,24}$, no se comprobaron diferencias significativas (estudios de tratamiento $1 a, 1 b y 2 b$ ).

\section{Retorno a actividad laboral}

Se localizaron 9 artículos en los que se comparó la variable "retorno a actividad habitual" (2 RS y 7 EC). Una $\mathrm{RS}^{14}$ y tres $\mathrm{EC}^{17,23,24}$, reportaron que los pacientes sometidos a HI con malla regresaron a sus actividades cotidianas antes que aquellos sometidos a HI sin malla (estudios de tratamiento $1 b, 2 a$ y $2 b$ ); de hecho el impacto de esta medición se ejemplifica en un $\mathrm{EC}^{24}$, en el que se determinó un promedio de retorno de $14 \pm 3$ días vs $25 \pm 7$ días, para HI con malla respecto de HI sin malla, con un valor de $p<0,05$ (estudios de tratamiento 1b). Por otra parte, una $\mathrm{RS}^{15}$ y un $\mathrm{EC}^{19}$, también reportaron un retorno a la actividad laboral en menor tiempo en el grupo de HI con malla, sin embargo, la diferencia no fue significativa (estudios de tratamiento 1 a $y$ 2b). En los tres $\mathrm{EC}$ restantes ${ }^{18,22,26}$, no se encontraron diferencias estadísticamente significativas entre ambas opciones de HI en relación a esta variable (estudios de tratamiento $2 b$ ).

\section{Costes involucrados}

Se localizaron 5 artículos en los que se comparó la variable "costes involucrados" (5 EC). Un $\mathrm{EC}^{24}$, que consideró en de la estimación, los costos iniciales de la cirugía y las complicaciones inmediatas, reportó un costo total menor para el grupo de HI con malla respecto de Shouldice (\$ 838 vs $\$ 885$ ), con un valor de $\mathrm{p}<0,05$ (estudios de tratamiento $1 b$ ). En los otros $4 \mathrm{EC}^{18,22,25,28}$, los costos totales asociados a la reparación quirúrgica fueron significativamente menores en el grupo de pacientes con $\mathrm{HI}$ sin malla (estudios de tratamiento $2 b$ ); por ejemplo, un $\mathrm{EC}^{22}$ reportó costos de $€ 235$ vs $€ 180(\mathrm{p}<0,01)$ y otro ${ }^{25}$, de 6.221 yuan vs 4.518 yuan $(\mathrm{p}<0,001)$ para HI con malla respecto de sin malla (estudios de tratamiento $2 b$ ).

\section{Dolor postoperatorio persistente}

Se localizaron 12 artículos en los que se comparó la variable "dolor postoperatorio persistente" (3 RS y 9 EC). Dos RS ${ }^{14,15}$, demostraron menor dolor crónico utilizando la reparación con malla; sin embargo, en uno de ellos ${ }^{14}$, la diferencia se explica por un EC multicéntrico de gran tamaño publicado en 1999 que demuestra una diferencia; pero si este se excluye del análisis el efecto estimado fue similar en ambos grupos (estudios de tratamiento 2a). En la otra $\mathrm{RS}^{15}$, el dolor persistente también fue menor en HI con malla (OR: 0,68 e IC 95\%: $0,47$ a 0,98$)$, sin embargo, este resultado fue dependiente de uno de los EC (Bietigheim 1998), el que reportaba altos porcentajes de dolor (estudios de tratamiento 1a). En $8 \mathrm{EC}^{18,20,21,23,25-28,30}$, no se objetivaron diferencias significativas; sin embargo, 
Uso vs no uso de malla en hernioplastía inguinal. Overview - C. Manterola et al

en uno de ellos ${ }^{18}$, se evidenció mayor consumo de analgésico en pacientes en los que se utilizó HI con técnica de Shouldice (estudios de tratamiento $1 b y$ $2 b$ ). Finalmente, una $\mathrm{RS}^{16}$, reveló evidencia a favor, aunque no significativa, de la técnica de Shouldice al compararla con HI con malla (OR: 0,87 e IC 95\%: 0,55 a 1,39), (estudios de tratamiento 1a).

\section{Recurrencia}

Se localizaron 11 artículos en los que se comparó la variable "recurrencia" (3 RS y 8 EC). En las tres $\mathrm{RS}^{14-16}$, se concluyó que la recurrencia fue significativamente menor en las técnicas de $\mathrm{HI}$ con malla (estudios de tratamiento $1 a$ y $2 a$ ). En una $\mathrm{RS}^{15}$, se verificó una reducción de $50 \%$ a $75 \%$ a favor de HI con malla (OR 0,37 e IC 95\%: 0,26 a 0,51 ); en otra ${ }^{16}$, la recurrencia fue mayor con la técnica de Shouldice vs Lichtenstein (OR: 3,80 e IC 95\%: 1,99 a 7,26). Por otra parte, 8 EC evaluaron la recurrencia, en 5 de los cuales se determinó menor recurrencia en las técnicas de $\mathrm{HI}$ con respecto de técnicas sin malla ${ }^{23,25,27-29}$; es así como el impacto se refleja en cifras de recurrencia de $2,8 \%$ vs $8,9 \%$ (p $=0,02)^{25}, 1,0 \%$ vs $7,0 \%(\mathrm{p}<0,001)^{28}$, para técnicas con malla respecto de HI sin malla; sin embargo, en otro de ellos ${ }^{23}$, la diferencia fue marginal $(\mathrm{p}=$ $0,08)$, con una reducción del riesgo absoluto de $2 \%$ luego de la HI con malla vs la reparación sin malla (estudios de tratamiento $1 b y 2 b$ ). Finalmente, en otros tres $\mathrm{EC}^{17,19,26}$, no se verificaron diferencias estadísticamente significativas (estudios de tratamiento $2 b$ ).

\section{Discusión}

A pesar que han transcurrido más de 120 años desde que Eduardo Bassini introdujo los conceptos modernos de la reparación de la hernia inguinal en 1887; el método estándar de la HI no ha variado de forma sustancial, pues las diversas técnicas existentes que se realizan por vía abierta se basan en los mismos principios; sin embargo, persisten discusiones respecto de que técnica tiene mejores resultados, incluyendo y mezclando en esta disputa técnicas de HI con aplicación de malla con técnicas de reparación convencional o sin utilización de mallas. Y, a pesar de ser la HI una de las intervenciones más frecuentes de realizar sólo hace tres años, la European Hernia Society (EHS) ${ }^{3}$ intentó normalizar esta situación y reducir a través de la publicación de una guía para el tratamiento de la hernia, la incertidumbre existente; pero a pesar de esto, la polémica entre grupos quirúrgicos persiste.

Este overview, permitió examinar el comportamiento de algunas variables al comparar mediante estudios del mejor nivel de evidencia disponible (RS y EC), entre "dos grupos" de técnicas de HI (con y sin malla). No obstante ello, se pudo objetivar que a pesar de tratarse de artículos de buen nivel de evidencia, estos son de calidad metodológica heterogénea y en ellos se comparan distintos tipos de técnicas (con y sin malla), a pesar que predomina la comparación entre HI de Lichtentein y Shouldice.

A pesar de todo, se logró objetivar, que en términos generales se puede comentar que las técnicas de $\mathrm{HI}$ con malla se asocian a menor tiempo quirúrgico, dolor postoperatorio, tiempo de hospitalización, retorno a la actividad habitual y recurrencia respecto de las técnicas de HI sin malla; y que existe cierta evidencia que soporta el hecho de que estas últimas, son menos costosas que las anteriores en términos simples; lo que se correlaciona con la literatura existente.

Respecto a lo anteriormente expuesto, vale la pena reflexionar lo expresado en la Tabla 1, que resume la evidencia encontrada en este estudio en términos de las variables consideradas, el número de estudios que soportan la evidencia de su análisis, la calidad de la evidencia y el resumen de los hallazgos. En relación a ella, es importante señalar que se acepta por calidad alta a aquella que emana de RS y EC sin limitaciones importantes; por lo que se asumen que es poco probable que estudios futuros cambien demasiado la estimación del efecto y por lo tanto, el grado de recomendación y la confianza en ellos es alta. Y, como calidad moderada a aquella que procede de RS y EC con limitaciones, defectos metodológicos o imprecisiones; por lo que es probable que estudios futuros puedan cambiar la confianza en la estimación del efecto reportado en ellos ${ }^{13}$.

Sin embargo, se ha de tener en cuenta las limitaciones de este estudio, entre las que cabe destacar el potencial sesgo de haber trabajado con sólo tres bases de datos (si bien es cierto que las utilizadas son aceptadas como de alta visibilidad, también se ha de considerar que existen otras en las que pudiesen existir RS y EC que obviamente no fueron consideradas en este estudio); de haber utilizado una estrategia de búsqueda restringida sólo a cierto 
Uso vs no uso de malla en hernioplastía inguinal. Overview - C. Manterola et al

tipo de diseños de investigación clínica(no se consideraron estudios de cohortes, casos y controles y series de casos; pues la intención fue trabajar con el mejor nivel de evidencia disponible); y de no haber examinado aquellos EC no incluidos en las RS identificadas (hecho complejo de resolver, pues la estrategia de búsqueda utilizada no arrojó más estudios que los que fueron finalmente analizados; a menos, claro está, que consideremos otras bases de datos distintas a las utilizadas).

Otro hecho de interés en esta revisión global de la evidencia, es que se requiere de mayor investigación en el área, en términos de evaluar resultados de HI con y sin malla en situaciones de hernias recidivadas, hernias bilaterales y situaciones de urgencia; valorar el rol de la HI laparoscópica en relación a técnicas abiertas (ya sea con o sin malla); determinar relación coste beneficio, coste efectividad o coste utilidad en HI con y sin malla; y por último valorar calidad de vida asociada a estos procedimientos.

\section{Referencias}

1. Primatesta P, Goldacre MJ. Inguinal hernia repair: incidence of elective and emergency surgery, readmission and mortality. Int J Epidemiol 1996; 25: 835-9.

2. Bay-Nielsen M, Kehlet H, Strand L, Malmstrøm J, Andersen FH, Wara P, et al. Quality assessment of 26,304 herniorrhaphies in Denmark: a prospective nationwide study. Lancet 2001; 358: 1124-8.

3. Simons MP, Aufenacker T, Bay-Nielsen M, Bouillot JL, Campanelli G, Conze J, et al. European Hernia Society guidelines on the treatment of inguinal hernia in adult patients. Hernia 2009; 13: 343-403.

4. Liem MS, van Vroonhoven TJ. Laparoscopic inguinal hernia repair. Br J Surg 1996; 83: 1197-204.

5. EU Hernia Trialists Collaboration. Mesh compared with non-mesh methods of open groin hernia repair: systematic review of randomized controlled trials. Br J Surg 2000; 87: 854-9.

6. Lichtenstein IL, Shulman AG, Amid PK, Montllor MM. The tension-free hernioplasty. Am J Surg 1989; 157: 18893.

7. Nordin P, Bartelmess P, Jansson C, Svensson C, Edlund G. Randomized trial of Lichtenstein versus Shouldice hernia repair in general surgical practice. Br J Surg 2002; 89: 45-9.

8. Manterola C, Pineda V, Tort M, Targarona E, Villegas Portero R, Alonso P. Effectiveness of Laparoscopic Sur- gery for Gallstones and Common Bile Duct Lithiasis: a Systematic Overview. Int J Morphol 2010; 28: 729-42.

9. Jadad AR, Moore RA, Carroll D, Jenkinson C, Reynolds DJ, Gavaghan DJ, et al. Assessing the quality of reports on randomized clinical trials: Is blinding necessary? Controlled Clin Trials 1996; 17: 1-12.

10. Escala de Jadad. http://33m.lista.cl/wiksocial/Escala_de_Jadad.html. Acceso el 2 de octubre de 2012.

11. Scottish Intercollegiate Guidelines Network (SIGN). Scottish Intercollegiate Guidelines Network. SIGN 50: a guideline developer's' handbook. Edinburgh, Revised edition, November 2011. http://www.sign.ac.uk/pdf/ sign50.pdf. Acceso el 3 de octubre de 2012.

12. Oxford Centre for Evidence-based Medicine (OCEBM). Levels of Evidence (March 2009). http://www.cebm.net/ index.aspx?o=1025. Acceso el 3 de octubre de 2012.

13. Guyatt G, Gutterman D, Baumann MH, AddrizzoHarris D, Hylek EM, Phillips B, et al. Grading strength of recommendations and quality of evidence in clinical guidelines: report from an American College of Chest Physicians Task Force. Chest 2006; 129: 174-81.

14. Grant AM; EU Hernia Trialists Collaboration. Open mesh versus non-mesh repair of groin hernia: metaanalysis of randomised trials based on individual patient data [corrected]. Hernia 2002; 6: 130-6.

15. Scott NW, McCormack K, Graham P, Go PM, Ross SJ, Grant AM. Open mesh versus non-mesh for repair of femoral and inguinal hernia. Cochrane Database Syst Rev 2002; (4): CD002197.

16. Amato B, Moja L, Panico S, Pérsico G, Rispoli C, Rocco $\mathrm{N}$, Moschetti I. Shouldice technique versus other open techniques for inguinal hernia repair. Cochrane Database Syst Rev. 2012 Apr 18; 4: CD001543.

17. Harjai M, Nagpal B, Singh P, Singh Y. A Prospective Randomized Controlled Study of Lichtenstein's Tension Free versus Modified Bassini Repair in the Management of Groin Hernias. Medical Journal Armed Forces India (MJAFI) 2007; 63: 40-3. http://medind.nic.in/maa/t07/ i1/maat07ilp40.pdf. Acceso el 20 de julio de 2012.

18. Damamme A, Samama G, D’Alche-Gautier MJ, Chanavel N, Bréfort JL, Le Roux Y. Medico-economic evaluation of treatment of inguinal hernia: Shouldice vs laparoscopy. Ann Chir 1998; 52: 11-6.

19. Kaynak B, Celik F, Guner A, Guler K, Kaya MA, Celik M. Moloney darn repair versus lichtenstein mesh hernioplasty for open inguinal hernia repair. Surg Today 2007; 37: 958-60.

20. Köninger J, Redecke J, Butters M. Chronic pain after hernia repair: a randomized trial comparing Shouldice, Lichtenstein and TAPP. Langenbecks Arch Surg 2004; 389: 361-5. 
21. Kucuk HF, Sikar HE, Kurt N, Uzun H, Eser M, Tutal F, et al. Lichtenstein or darn procedure in inguinal hernia repair: a prospective randomized comparative study. Hernia 2010; 14: 357-60.

22. Porrero JL, Bonachía O, López-Buenadicha A, Sanjuanbenito A, Sánchez-Cabezudo C. Repair of primary inguinal hernia: Lichtenstein versus Shouldice techniques. Prospective randomized study of pain and hospital costs. Cir Esp 2005; 77: 75-8.

23. Prieto-Díaz-Chávez E, Medina-Chávez JL, GonzálezOjeda A, Coll-Cárdenas R, Uribarren-Berrueta O, Trujillo-Hernández B, et al. Tension-free hernioplasty versus conventional hernioplasty for inguinal hernia repair. Surg Today 2005; 35: 1047-53.

24. Prieto-Díaz-Chávez E, Medina-Chávez JL, Anaya-Prado R. A cost-effectiveness analysis of tension-free versus shouldice inguinal hernia repair: a randomized doubleblind clinical trial. Hernia 2009; 13: 233-8.

25. Shi Y, Su Z, Li L, Liu H, Jing C. Comparing the effects of Bassini versus tension-free hernioplasty: 3 years' followup. Front Med China 2010; 4: 463-8.
26. Szopinski J, Dabrowiecki S, Pierscinski S, Jackowski M, Jaworski M, Szuflet Z. Desarda versus Lichtenstein technique for primary inguinal hernia treatment: 3-year results of a randomized clinical trial. World J Surg 2012; 36: 984-92.

27. van Veen RN, Wijsmuller AR, Vrijland WW, Hop WC, Lange JF, Jeekel J. Randomized clinical trial of mesh versus non-mesh primary inguinal hernia repair: longterm chronic pain at 10 years. Surgery 2007; 142: 695-8.

28. Vrijland WW, van den Tol MP, Luijendijk RW, Hop WC, Busschbach JJ, de Lange DC, et al. Randomized clinical trial of non-mesh vs. mesh repair of primary inguinal hernia. Br J Surg 2002; 89: 293-7.

29. Elsebae MM, Nasr M, Said M. Tension-free repair versus Bassini technique for strangulated inguinal hernia: A controlled randomized study. Int J Surg. 2008 Aug; 6 (4): 302-5.

30. Bay-Nielsen M, Nilsson E, Nordin P, Kehlet H; Swedish Hernia Data Base the Danish Hernia Data Base. Chronic pain after open mesh and sutured repair of indirect inguinal hernia in young males. Br J Surg 2004; 91: 1372-6. 